\title{
Hydro-reservoir management and unit-commitment in energy optimization
}

\author{
Flávio leite loução Jr. and Marlon Sproesser mathias and Claudia \\ Sagastizábal and Luiz-Rafael Santos and Francisco Nogueira Calmon \\ SOBRAL
${ }^{1}$ Escola de Engenharia de São Carlos, Universidade de São Paulo (USP) Brazil
${ }^{2}$ Universidade Estadual Campinas (UNICAMP), Brazil
${ }^{3}$ Radix Engenharia, Brazil
${ }^{4}$ Universidade Estadual de Maringá (UEM), Brazil
${ }^{4}$ Universidade Federal de Santa Catarina (UFSC), Brazil

(Communicated to MIIR on 3 September 2021)

Study Group: 6th Brazilian Study Group with Industry, 22-26 March 2021, São Carlos

Communicated by: Francisco Louzada Neto and Jose Alberto Cuminato

Industrial Partner: Radix Engenharia

Presenter: Flávio Leite Loução Junior

Team Members: Claudia Sagastizábal (Universidade Estadual de Campinas), Paulo J. S. Silva (Universidade Estadual de Campinas), Juan Pablo Luna (Universidade Federal do Rio de Janeiro), Flávio Leite Loução Junior (Radix), Marlon Sproesser Mathias (Escola de Engenharia de São Carlos, Universidade de São Paulo) Francisco Nogueira Calmon Sobral (Universidade Estadual de Maringá), Luiz-Rafael Santos (Universidade Federal de Santa Catarina), Marcelo Cordova (Universidade Federal de Santa Catarina), Petra Bartmeyer (Instituto de Ciências Matemáticas e de Computação, Universidade de São Paulo), Lucio Vargas de Albuquerque Nunes (Universidade Federal de Lavras), Otavio Carvalho (Universidade Federal do Maranhão) and Larissa Tebaldi de Oliveira (Instituto de Ciências Matemáticas e de Computação, Universidade de São Paulo)

Industrial Sector: Energy/Utilities

Tools: Mathematical optimization, Julia, Python

Key Words: hydro-unit-commitment, reservoir management, mathematical optimization

MSC2020 Codes: 49M29, 90C90 


\section{Summary}

In partnership with CCEE, CEPEL and RADIX as industrial partners, in 2021 the study group focused on the dynamics of hourly prices when industrial consumers are demand responsive, as a follow-up of the industrial problem tackled in 2018 and 2019, on "Day-ahead pricing mechanisms for hydro-thermal power systems". Demand response is currently being tested by the Brazilian independent system operator and by the trading chamber, ONS. The program considers reductions of consumption of some clients as an alternative to dispatching thermal power plants out of the merit order. The day-ahead problem of finding optimal dispatch and prices for the Brazilian system is modelled as a mixed-integer linear programming problem, with non-convexities related to fixed costs and minimal generation requirements for some thermal power plants. The work focuses on the point of view of an individual hydro-power generator, to determine business opportunities related to adhering to a demand response program.

\section{Introduction}

Due to the extensive territorial area of the country and the existence of a series of plateaus and mighty rivers, Brazil has hydroelectricity as its main source of energy generation. From an operational point of view, hydroelectric generation plants are flexible resources that make it possible to regulate the amount of energy generated, as well as the levels of reservoirs and rivers present in their areas of influence [2]. In this context, it is important to optimize the operation of hydroelectric plants regarding both the operating costs and the generation of revenue [5].

The operational optimization of a hydroelectric power plant consists in determining an optimal energy production planning for a series of generating units, considering a range of technical, physical and strategic constraints. The difficulties related to this problem arise not only from the non-linear aspects involved, but also from the need to obtain the optimal solution in a short period of time [5]. Thus, this study addresses the problem of optimal allocation of hydroelectric units in the context of cascading power plants operation, based on the work developed by Scuzziato [4], with a contribution towards considering the operator's profit in the optimization problem. In Cristian Finardi and Reolon Scuzziato [1] and Scuzziato [4], the profit is measured by the amount of water that is kept in the reservoirs and by the reduction of unnecessary usage of units.

The approach used is based on the incorporation of economic variables to the mathematical formulation of the problem, considering a more flexible energy demand that allows the operator, in possession of a market forecast for the considered time horizon, to concentrate production in periods where this is more economically advantageous. Future costs were modelled considering the volume of water present in the reservoirs, measured at the end of each period, since this is the plant's asset, and it is desirable to have reservoirs with more water at times when this is more economically advantageous. The solution strategy adopted is to face the problem from a linear and non-linear point of view, aiming to establish a comparative basis between both formulations. Furthermore, 
the restrictions adopted are based on those indicated by Scuzziato [4], with the difference that some parameters previously modelled by fourth degree polynomials were expressed in quadratic formulations, aiming to improve the computational performance.

\section{Problem description}

In this section, we present the modelling of the mathematical programming problem of multiple stages of generating units, which considers the operation of hydroelectric plants in cascade, disregarding the existence of prohibited zones in the hills curves. This approach differs from the ones presented by Gomes [3] and Scuzziato [4], since it requires the use of 0-1 variables. In what follows, units are in the SI, except for volumes, given in $\mathrm{hm}^{3}$ and powers, given in MW.

The sets, indexes and parameters are:

- $R$ : total reservoirs $(r \in\{1, \ldots, R\})$,

- $T$ : total planning stages $(t \in\{1, \ldots, T\})$,

- $n_{r t}$ : total generating units in reservoir $r$ at time $t\left(j \in\left\{1, \ldots, n_{r t}\right\}\right)$,

- Rup : total reservoirs upstream of reservoir $r$,

- $\sigma_{j r}$ : start up or shut down cost of unit $j$ of reservoir $r$,

- $L_{r t}$ : power demanded by the system from reservoir $r$ at time step $t$,

- $\bar{c}$ : proportionality constant between flow and volume,

- $y_{r t}$ : incremental flow affluent to the reservoir $r$ in the time step $t$,

- $\tau_{m r}$ : water displacement time delay between reservoirs $m$ and $r$,

- $v_{r}^{\min }, v_{r}^{\max }$ : minimum and maximum volumes of reservoir $r$,

- $p g_{j r}^{\min }, p g_{j r}^{\max }$ : minimum and maximum power output by unit $j$ of reservoir $r$,

- $g$ : gravitational acceleration,

- $a_{i}$ : upstream quota polynomial coefficients, where $i=\{0,1,2,3,4\}$,

- $b_{i}$ : downstream quota polynomial coefficients, where $i=\{0,1,2,3,4\}$,

- $c_{i}$ : efficiency curve coefficients, where $i=\{0,1,2,3,4,5\}$,

- $d_{i}$ : minimum flow coefficients, where $i=\{0,1,2,3\}$,

- $e_{i}$ : maximum flow coefficients, where $i=\{0,1,2,3\}$,

- $G$ : constant related to the specific weight of water and unit conversions, equals to $9,81 \times 10^{-3}$

- $k s_{j r}$ : proportionality constant for the hydraulic losses of unit $j$ of reservoir $r$,

- $k p_{j r}$ : proportionality constant for friction losses in the individualized ducts of unit $j$ of reservoir $r$,

- $k p u_{r}$ : proportionality constant for friction losses in the duct common to all units of reservoir $r$,

- $\pi_{t}$ : price of energy at time $t$.

The variables are described as follows:

- $q_{j r t}$ : turbine flow through unit $j$ of reservoir $r$ at time $t$,

- $Q_{r t}$ : turbine flow in reservoir $r$ at time $t$,

- $s_{r t}$ : flow discharged from reservoir $r$ at time $t$,

- $u_{j r t}$ : boolean that indicates whether unit $j$ of reservoir $r$ at time $t$ is on or off, 
- $p g_{j r t}$ : power generated by unit $j$ of reservoir $r$ at time $t$,

- $v_{r t}$ : volume of reservoir $r$ at time $t$,

- $q_{j r t}^{\min }, q_{j r t}^{\max }$, minimum and maximum turbine flow of unit $j$ of reservoir $r$ at time $t$,

- $h b_{r t}$ : gross water drop of reservoir $r$ at time $t$,

- $f_{c m}$ : water elevation level upstream of reservoir $r$ at time $t$,

- $f c j_{r t}$ : water elevation level downstream of reservoir $r$ at time $t$,

- pst $_{j r t}$ : output power of unit $j$ of reservoir $r$ at time $t$,

- $\eta_{j r t}$ : efficiency of unit $j$ of reservoir $r$ at time $t$,

- $h_{j r t}$ : net water drop of unit $j$ of reservoir $r$ at time $t$,

- $h l s_{j r t}$ : gross drop attenuation factor due to hydraulic losses of unit $j$ of reservoir $r$ at time $t$,

- $h l p_{j r t}$ : gross drop attenuation factor due to water friction in the duct of unit $j$ of reservoir $r$ at time $t$,

- $p e g_{j r t}$ : power delivered by unit $j$ of reservoir $r$ at time $t$,

- $p_{\text {jrt }}$ : mechanical losses in unit $j$ of reservoir $r$ at time $t$,

- $p g g_{j r t}$ : global losses in unit $j$ of reservoir $r$ at time $t$.

The constraints used to model the cascaded reservoir problem are given next. We observe that, depending on the approach, some of them are used and others are not, as 
will be detailed in Section 3.

$$
\begin{aligned}
& \sum_{j=1}^{n_{r t}} p g_{j r t} \geq L_{r t} \\
& v_{r t}=v_{r(t-1)}+\bar{c} y_{r t}-\bar{c}\left[Q_{r t}+s_{r t}-\sum_{m=1}^{R u p_{r}}\left(Q_{r\left(t-\tau_{m r}\right)}+s_{r\left(t-\tau_{m r}\right)}\right)\right] \\
& Q_{r t}=\sum_{j=1}^{n_{r t}} q_{j r t} \\
& p g_{j r t}-p s t_{j r t}\left(v_{r t}, Q_{r t}, q_{j r t}, s_{r t}\right)+p m t_{j r t}\left(p g_{j r t}\right)+p g g_{j r t}\left(p g_{j r t}\right)=0 \\
& v_{r}^{\min } \leq v_{r t} \leq v_{r}^{\max } \\
& q_{\text {jrt }}^{\min } \leq q_{\text {jrt }} \leq q_{\text {jrt }}^{\max } \\
& p g_{j r}^{\text {min }} u_{j r t} \leq p g_{j r t} \leq p g_{j r}^{\max } u_{j r t} \\
& f c m_{r t}=a_{0}+a_{1} v_{r t}+a_{2} v_{r t}^{2}+a_{3} v_{r t}^{3}+a_{4} v_{r t}^{4} \\
& f c j_{r t}=b_{0}+b_{1}\left(Q_{r t}+s_{r t}\right)+b_{2}\left(Q_{r t}+s_{r t}\right)^{2}+b_{3}\left(Q_{r t}+s_{r t}\right)^{3}+b_{4}\left(Q_{r t}+s_{r t}\right)^{4} \\
& h b_{r t}=f c m_{r t}-f c j_{r t} \\
& h l s_{j r t}=k s_{j r} q_{j r t}^{2} \\
& h l p_{j r t}=k p_{j r} q_{j r t}^{2}+k p u_{r} Q_{r t}^{2} \\
& h_{j r t}=h b_{r t}-h l p_{j r t}-h l s_{j r t} \\
& \eta_{j r t}=c_{0}+c_{1} q_{j r t}+c_{2} h_{j r t}^{2}+c_{3} h_{j r t}^{3}+c_{4} h_{j r t}^{4}+c_{5} h_{j r t}^{4} \\
& p m t_{j r t}=g_{0}+g_{1} p g_{j r t}+g_{2} p g_{j r t}^{2} \\
& p g g_{j r t}=f_{0} e^{f_{1} p g_{j r t}} \\
& p s t_{j r t}=G \eta_{j r t} h_{j r t} q_{j r t} \\
& \text { peg }_{j r t}=\text { pst }_{j r t}-p m t_{j r t} \\
& p g_{j r t}=p e g_{j r t}-p g g_{j r t} \\
& q_{j r t}^{\min }=d_{0}+d_{1} h_{j r t}+d_{2} h_{j r t}+d_{3} h_{j r t}^{2} \\
& q_{j r t}^{\max }=e_{0}+e_{1} h_{j r t}+e_{2} h_{j r t}+e_{3} h_{j r t}^{2} \\
& Q_{r t} \geq 0, \quad s_{r t} \geq 0, \quad v_{r t} \geq 0 \\
& h b_{r t} \geq 0, \quad f c m_{r t} \geq 0, \quad f c j_{r t} \geq 0, \quad h_{r t} \geq 0 \\
& h l p_{r t} \geq 0, \quad h l s_{r t} \geq 0, \quad q_{r t}^{\min } \geq 0, \quad q_{r t}^{\max } \geq 0 \\
& p g_{j r t} \geq 0, \quad q_{j r t} \geq 0, \quad p s t_{j r t} \geq 0, \quad \eta_{j r t} \geq 0 \\
& p e g_{j r t} \geq 0, \quad p m t_{j r t} \geq 0, \quad p g g_{j r t} \geq 0 \\
& u_{\text {jrt }} \in\{0,1\}
\end{aligned}
$$

Index $j$ refers to each unit in a plant and is in range $j \in\left\{1, \ldots, n_{r t}\right\}$ while index $r$ refers to each plant in the system and is in range $r \in\{1, \ldots, R\}$. Finally, index $t$ refers to each time step and is in range $t \in\{1, \ldots, T\}$.

The constraints (2.1)-(2.27) describe the water flow along with the starting and stopping weights of units, satisfying operational constraints. Constraints (2.1) ensure that the demand is met, constraint (2.2) are responsible for the balance between the reser- 
voirs, constraint (2.3) compute the generation of each reservoir in each time step and constraints (2.4) are the non-linear terms relative to the production units.

Constraints (2.5) ensure that the volume of reservoirs is within the minimum and maximum operating limits, constraint (2.6) guarantee the operating flow limits, while constraint (2.7) certify the observance of power generation limits per unit.

Constraints (2.8)-(2.13) relate to the water drop. Constraint (2.14) is the hydraulic efficiency of the turbine, constraint (2.15) relates to the mechanical losses in the turbine, constraint (2.16) are related to global losses in the generator and constraints (2.17)-(2.19) are related to the overall power output. Constraints (2.20)-(2.21) compute the flow limits and, finally, constraints (2.22)-(2.27) define the domain of the variables.

As already mentioned, two approaches were used: mixed-integer linear programming and nonlinear programming. For each approach, some constraints (2.1)-(2.27) have to be discarded or approximated. Details about the constraints used and their relaxation are the subject of Section 3. A key difference between each approach is the objective function of the optimization problem. In the mixed-integer linear programming approach, the goal is to minimize amount of water used to satisfy demand and also the number of times a unit is switched on and off for each reservoir in the cascade, which can be written as

$$
\min \sum_{t=1}^{T} \sum_{r=1}^{R}\left[Q_{r t}+s_{r t}+\sum_{j=1}^{n_{r t}} \sigma_{j r}\left[u_{j r t}\left(1-u_{j r(t-1)}\right)+u_{j r(t-1)}\left(1-u_{j r t}\right)\right]\right] .
$$

In the nonlinear programming approach, the forecast prices are taken into consideration, while the binary variables are dropped. Therefore, the objective function is just to maximize the profit when selling energy

$$
\max \sum_{t=1}^{T} \sum_{r=1}^{R} \sum_{j=1}^{n_{r t}} \pi_{t} p g_{j r t} .
$$

Clearly, such objective makes no sense in the case where demand has to be satisfied exactly. However, it is well known that cascaded reservoirs have more flexibility to satisfy demands. Usually, such demand constraints (2.1) are subject to agreements one day before or even can be satisfied by the whole cascade, instead of by each reservoir.

\section{Methodology and implementation}

Two types of implementations were considered for this problem. A non-linear model was developed, as shown in Section 3.1. For simplicity, this model disregards the integer optimization variables. In parallel, a Mixed Integer Linear Programming model was also implemented, it is shown in Section 3.2.

A library for reading and storing the data was also developed. It is described in Section 3.3 and is available at https://github.com/petrabartmeyer/6BSGI, along with the non-linear optimization routines. 


\subsection{Non-linear implementation}

The main objective of this study is to consider the profitability of the generated energy and to study the behaviour of the model when the considered objective changes to the price paid by the National System Operator (ONS, in Portuguese) for the generated power. Due to the complexity of the model and the short implementation and testing time, an initial simplification was considered, in which the binary variable which indicates whether unit $j$ of reservoir $r$ at time $t$ is on or off $\left(u_{j r t}\right)$ was disregarded. Due to this simplification, an additional constraint on the minimum final volume necessary at the end of the time horizon was added, to avoid reservoir depletion.

In order to help the solution of the model, a new variable was added, which represents the amount of energy that has to be bought in the spot market, to satisfy demand:

- $m c d_{r t}$ : power purchased by reservoir $r$ at time $t$ to meet demand,

The simplified model considered is given by:

Objective (2.29)

s. t.:

Constraints (2.2)-(2.6) and (2.8)-(2.27),

$\sum_{j=1}^{n_{r t}} p g_{j r t}+m c d_{r t} \geq L_{r t}, \quad r \in\{1, \ldots, R\}, t \in\{1, \ldots, T\}$,

$p g_{j r}^{\min } \leq p g_{j r t} \leq p q_{j r}^{\max }, \quad j \in\left\{1, \ldots, n_{r t}\right\}, r \in\{1, \ldots, R\}, t \in\{1, \ldots, T\}$,

$f c m_{r T} \geq v_{r}^{\text {final }}, \quad r \in\{1, \ldots, R\}$.

As mentioned, in this simplification we aim to maximize the profitability of the generated energy. Constraint (3.1) ensures that demand is met even if production is insufficient, while constraint (3.2) is a simplified version of (2.7), disregarding the boolean $u_{j r t}$. In the latter, we assume that all generating units are always on and respecting the minimum and maximum generation power. By avoiding losses due to turning generating units on or off and the pricing of the future value of water, we are avoiding artificial terms in the objective function, which could bring difficulties for correct pricing.

Finally, constraint (3.3) was added to ensure a minimum volume at the end of the period at each plant. This set of restrictions prevents the plant from being emptied at the end of the period, since, if there was no future cost for water, the model would benefit from using all the water in the reservoir. In addition, this set of restrictions is in accordance with current operation planning techniques, where the proposed load curve is drawn according to the level at which the reservoir is to be operated in the next planning horizon.

\subsubsection{Possible restrictions to ensure a generating unit turned on during consecutive periods}

It is not in the interest of the plants that units are switched on and off frequently. Once unit is turned on, it must remain on for a period of time. One of the challenges posed was to design a constraint capable of ensuring such behaviour. 
Consider $n_{t}^{o n}$ as the number of time units (for example, hours) that unit must remain on after being started and the following constraint based on binary variables $u_{j r t}$ :

$n_{t}^{o n}\left(u_{j r t_{0}}-u_{j r\left(t_{0}-1\right)}\right) \leq \sum_{t=t_{0}}^{t_{0}+n_{t}^{o n}} u_{j r t}, \quad j \in\left\{1, \ldots, n_{r t}\right\}, r \in\{1, \ldots, R\}, t_{0} \in\left\{3, \ldots, T-n_{t}^{o n}\right\}$.

This constraint guarantees that whenever a unit $j$ is activated, that is, $u_{j r t_{0}}-u_{j r\left(t_{0}-1\right)}=$ 1 , it will remain in operation for $n_{t}^{o n}$ time units. In the other cases, that is, $u_{j r t_{0}}-$ $u_{j r\left(t_{0}-1\right)}=0$ or -1 , this constraint will be released.

Due to the combinatorial nature imposed by the $u_{j r t}$ variables, these constraints were remodelled using the approach below.

As the idea of turning a generating unit on and off in a daily planning period is not a natural action, the set of constraints above has been replaced by a set of constraints that prevent large changes in generated power between two consecutive time periods. For this, a parameter $\delta \in[0,1]$ is used to control the percentage of the total power range $\left(p g^{\max }-p g^{\min }\right)$, which can be added or removed at each time interval. The new set of constraints is as follows:

$$
\begin{aligned}
& p g_{j r t}-p g_{j r(t+1)} \leq \delta\left(p g^{\max }-p g^{\min }\right), \forall j=1, \ldots, n_{r t}, r=1, \ldots, R, t=1, \ldots, T-1 \\
& p g_{j r(t+1)}-p g_{j r t} \leq \delta\left(p g^{\max }-p g^{\min }\right), \forall j=1, \ldots, n_{r t}, r=1, \ldots, R, t=1, \ldots, T-1
\end{aligned}
$$

Note that the use of both sets of constraints makes it possible to avoid using the nonlinear modulus equation.

\subsubsection{High-order constraints}

The set of constraints in (2.1)-(2.27) presents non-linear functions to model the losses considered in the model. Due to the non-convexity generated by these functions, the possibility of an exact solution is limited to small examples, in order to solve larger problems it is necessary to represent these functions in a linear or quadratic way. To convert sets of polynomial constraints of order greater than two, the techniques described in this section were used.

The upstream and downstream level functions ( $f c m$ and $f c j$, respectively) are given by quartic curves and the minimum and maximum flow values $\left(q^{\min }\right.$ and $\left.q^{\max }\right)$, by cubic curves. These curves are approximated by parabolas. Two approaches were implemented. The first is to use a Taylor polynomial from the midpoint of the range of possible values for the input of each function. The second is the interpolation by a parabola that passes through the starting, central and ending points of each interval. Figure 1 shows the value of the upstream quota function for each of the four plants in the model. There is no visible difference when using the original quartic polynomial or one of the quadratic approximations. A similar behaviour can be observed in the other three variables.

Using data brought by Scuzziato[4], the largest relative error of the Taylor polynomial approximation was $0.5 \%$ and occurred in a maximum flow curve. By using parabola interpolation, the maximum error was reduced to $0.18 \%$. The mean error, considering all variables, was $0.02 \%$ in both cases. Figure 2 illustrates the errors of each approximation for plant number 1 in the database. Taylor's approximation has its errors concentrated 


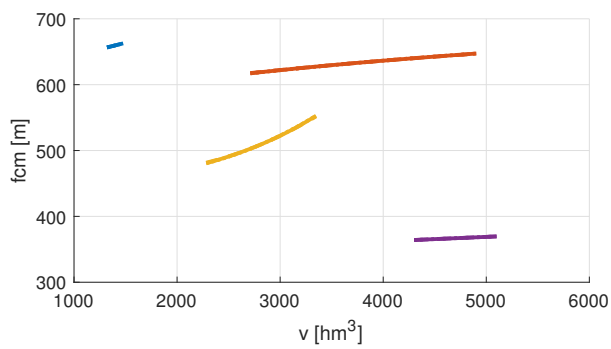

Figure 1. Value of the upstream quota function for the four plants in the model

at the extremities of the interval, while the interpolation has a more uniform distribution of errors.
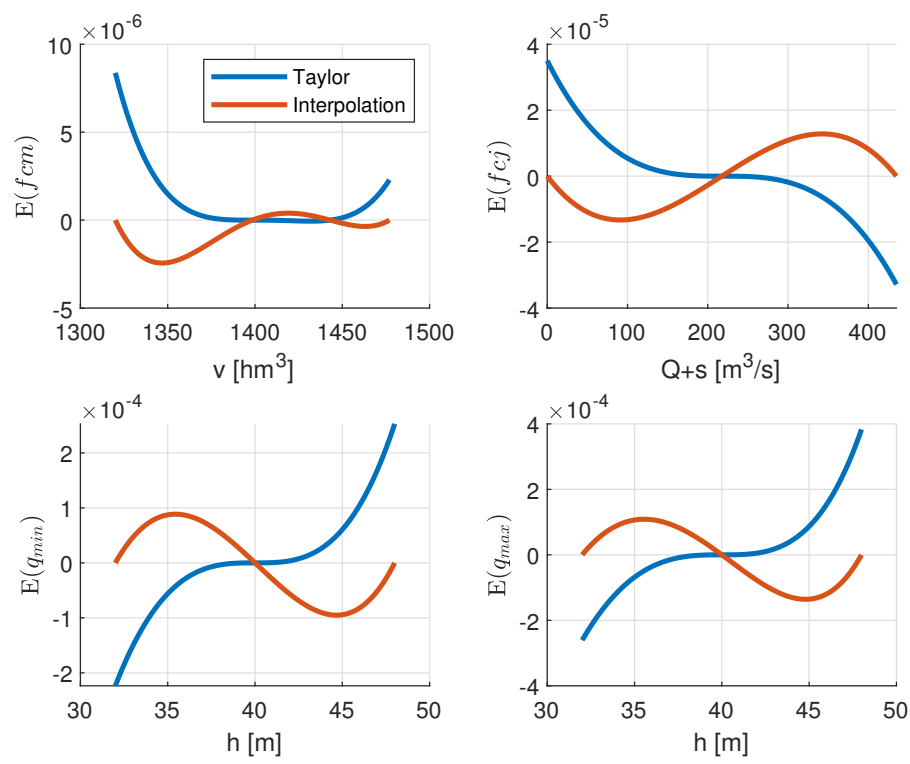

Figure 2. Relative errors in the approximation of cubic and quartic functions by quadratics

A similar methodology was used to compute the global losses in each generator ( $p g g)$, which are approximated by an exponential function. However, for the purposes of model simplification, a first order Taylor polynomial was used, its maximum error is less than $0.1 \%$ relative to the total generator power.

The hydraulic efficiency function $(\rho)$ of the turbine needed special attention. This is a bi-quadratic function, with input variables, net drop $(h)$ and turbine flow $(Q)$, whose result is again multiplied by $h$ and $Q$ to obtain the output power of the turbine (pst), resulting in a bi-cubic function. Auxiliary variables, $h 2=h^{2}, q 2=q^{2}$ e $h q$, were defined, which correspond to the product of the original variables. Thus, it was possible to reduce this bi-cubic equation to a system of quadratic or lower order equations. 


\subsection{Mixed-integer linear programming implementation}

Apart from the non-linear implementation described above, we have also implemented a linearised model which included the boolean restrictions. There is a considerable computational cost penalty due to the integer variables in the model, therefore, the whole model was linearised to keep the overall cost and complexity in a feasible range.

Nonetheless, some functions had to be linearised by parts, so that the overall error was not too large. As an example, Figure 3 compares the non-linear function that describes the power output of one generation unit with its linearised by parts' counterpart.

It is important to note that adding more segments to the linearisation quickly increases the number of constraints in the model, adding to the overall complexity and computational cost. In our test scenario, the fully linearised system had a total of 336 constraints. By using a two by two grid, as shown in Figure 3, this number is increased to 1344 constraints. A three by three grid further increases it to 3024 .

\subsection{Database description}

\subsubsection{Test scenarios}

Some test problems were created based on real instances of the National System. The data is organized by plant groups and, within a group, some instances are available. To access such data, a Python library was created. In Section 3.3.2 we describe the available tests and, in Section 3.3.3, how to access them through the Python library, which was also adapted for use in Julia.

\subsubsection{Problem description}

In this section, we describe the test problems that are available.

- p1 This problem represents a group of plants in cascade, given by the Campos Novos, Barra Grande, Machadinho and Itá plants, as can be seen in Figure 4. Data for this group were extracted from the work of Scuzziato [4]. It has the following instances:

- i1: no demand, hourly prices taken from the $23^{\text {rd }}$ of March 2021

○ i2: identical to i1, but with non-null demand. These demands were obtained from the Iguaçu hydrographic basin and are completely fictitious.

- p2 Cascading power plants on the Iguaçu River, illustrated in Figure 5. Composed of information from the plants (in order) Foz do Areia, Segredo, Salto Santiago, Salto Osório and Salto Caxias.

- p3 only the Itá plant (named H4) from data set p1. Its instances are:

$\circ$ i1: same as i1 from data set p1.

$\circ$ i2: same as i2 from data set $\mathbf{p} \mathbf{1}$ 


\subsubsection{Use of the library}

To use the library, it is currently necessary to access the data directory and then load it. The library has two functions:

- load_data(dataset): loads the information of the plants from this data set (given by a string). The result is a dict-type object with information about each plant and also specific information about each turbine.

- load_instance(dataset, instance, problem): loads information from an instance of the data set. The first and second arguments are strings. The third argument is the dictionary returned by the load_data call.

The concept behind this structure is that a group of plants can contain multiple instances with different values for testing. In practice, instances are subdirectories of the directories given by the data sets. Within such directories are a series of csv files whose filenames and column names cannot be modified. Furthermore, it is important that the names of the plants are consistent and always in the same order, to avoid problems in loading them. 

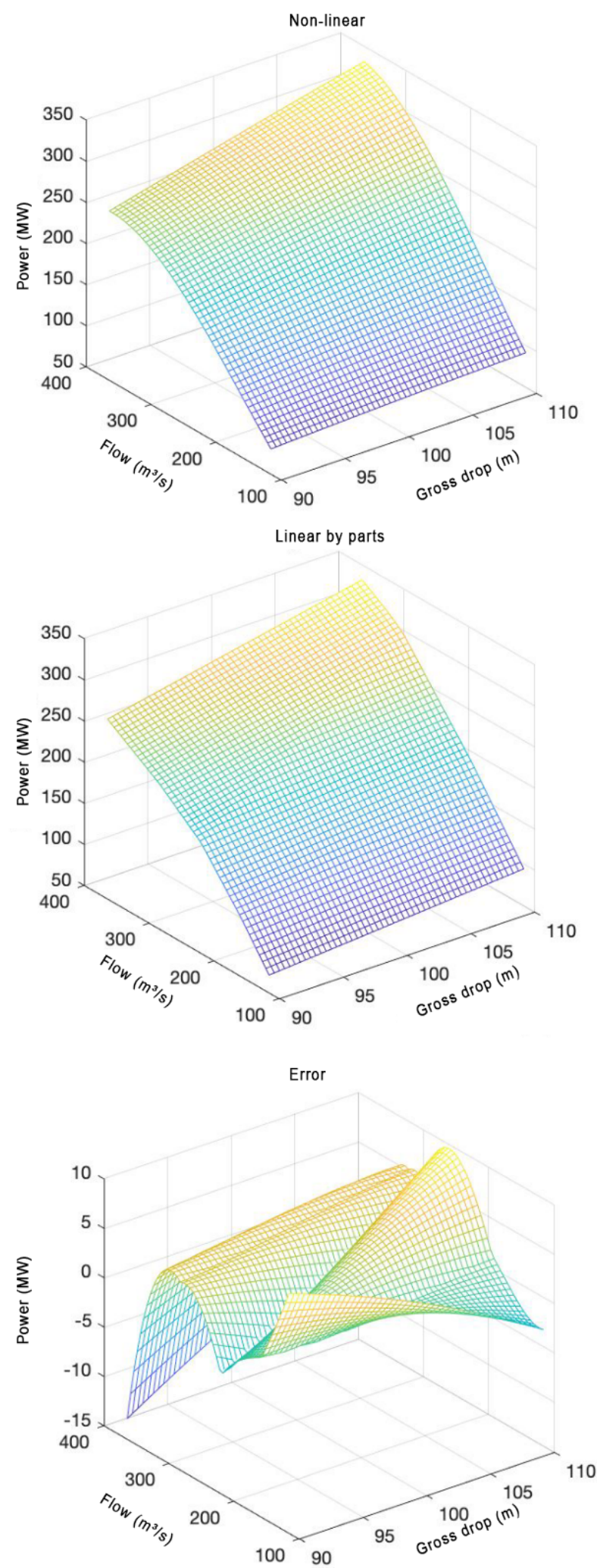

Figure 3. Power output by one generating unit. (top) Non-linear function. (centre) 2x2 Linear by parts. (bottom) Error. 


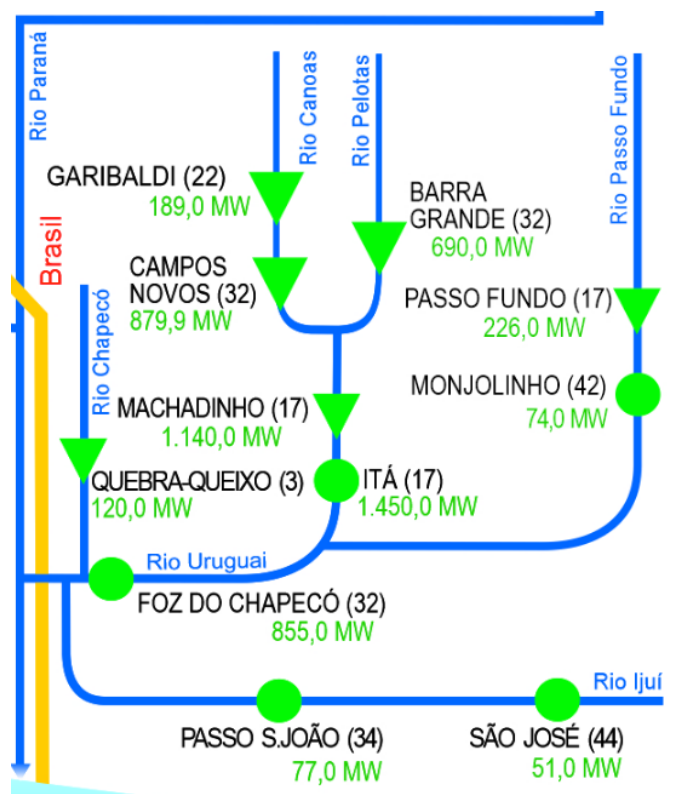

Figure 4. Illustration of the plants considered in the Uruguay basin.

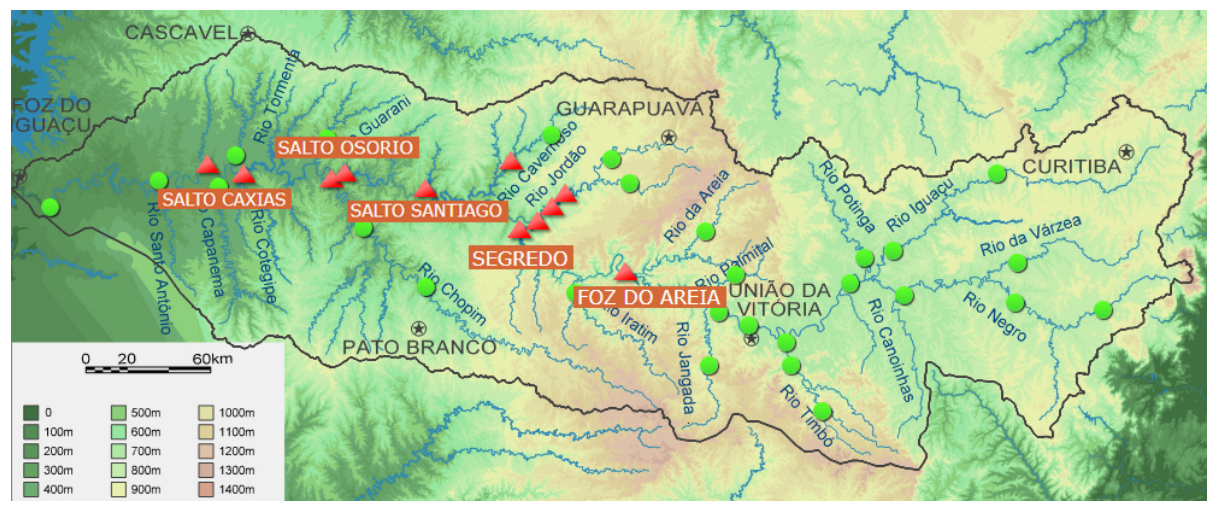

Figure 5. Illustration of the plants considered in the Iguaçu basin. 


\section{Results}

\subsection{Studies using the non-linear model}

The optimization model was implemented in Julia language using the JuMP package and the Gurobi solver (v9.1.1). The computational tests were performed on an Intel Xeon W2133 processor, in an Ubuntu 20.04 environment. The choice of the solver is due to its ability to solve non-convex quadratic optimization problems.

Two case studies were carried out. The first analyses the influence of the final level of the reservoir for the solution of the problem. The second case study seeks to propose demand curves that maximize the profit given by the power generated in each time step.

\subsubsection{Case study}

The scenario considered has 5 reservoirs with a variable number of turbines. The topology of connection between reservoirs and power is given by Figure 6. Time intervals of two hours were considered for water to reach from one plant to the next.

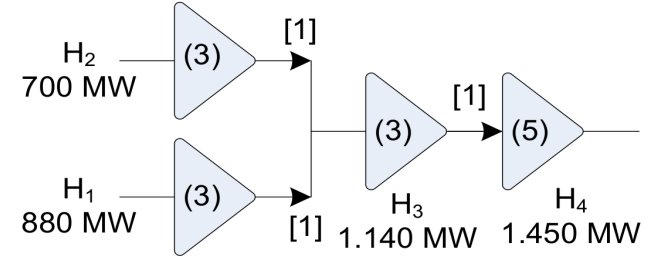

Figure 6. Scenario considered in the case study.

A 24-hour time span was set, with hourly varying prices per MW, shown in Figure 7. The demand curves for each of the plants are shown in Figure 8.

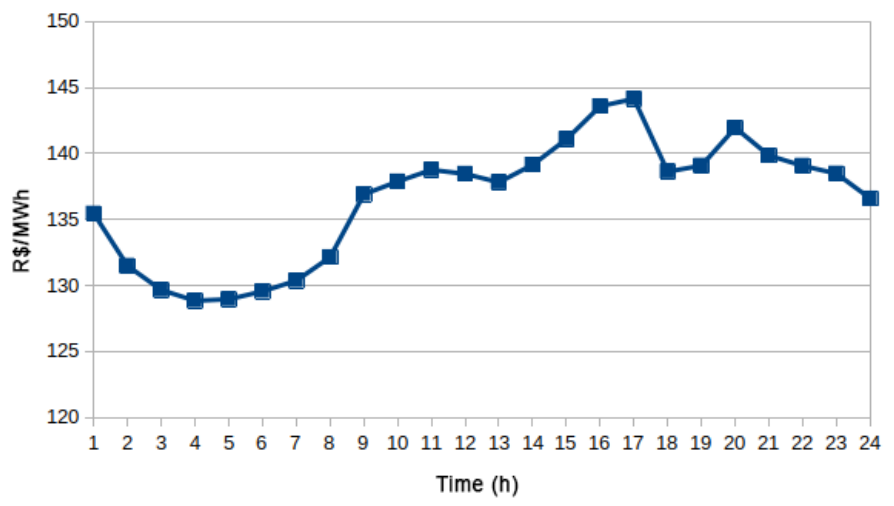

Figure 7. MWh price variation in each hourly interval. 


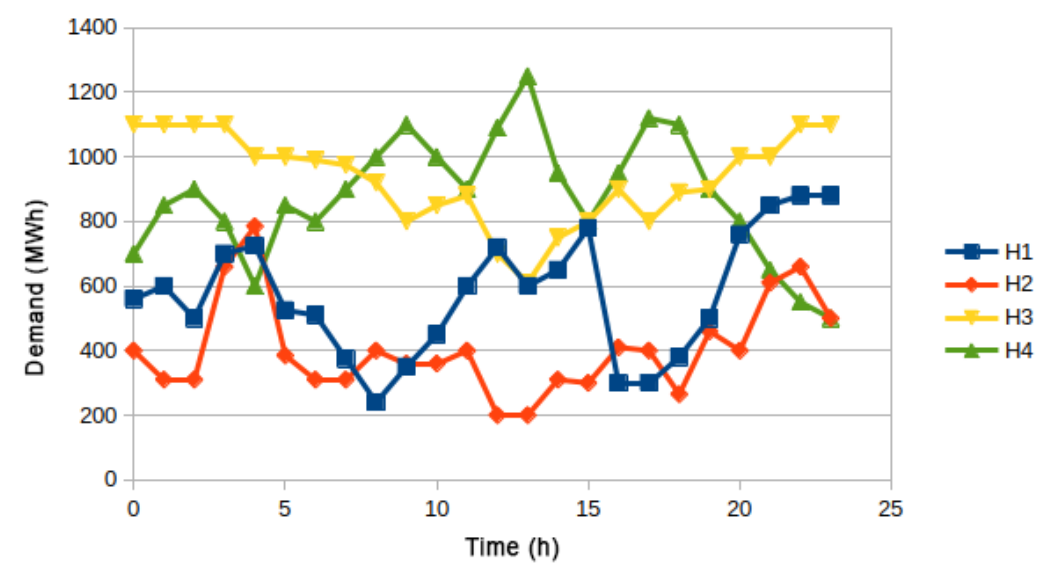

Figure 8. Demand of each plant by hourly period.

\subsubsection{Suggestion of the demand curve}

To create a demand suggestion curve, constraint (2.1) will be modified to receive a parameter $\alpha_{d} \in[0,1]$ which multiplies $L_{r t}$. Parameter $\alpha_{d}$ controls how much of the hourly demand must be met, for cases where the minimum cost of executing a demand curve is sought, the value of this parameter may be taken as $95 \%\left(\alpha_{d}=0.95\right)$, for example, which allows a $5 \%$ margin above or below the original demand curve. For cases where this demand curve must be proposed, the parameter value is taken as zero, allowing much more flexibility in the execution of the programmed load.

Three cases were considered: The first case allows executing powers generated in the interval between 0 and 2 times the nominal demand for that time interval $\left(\alpha_{d}=0\right)$, for the second interval the demand to be served is between 0.5 and 1.5 times the nominal value of demand $\left(\alpha_{d}=0.5\right)$, and the last case the demand met must be between 0.95 and 1.05 of the nominal value $\left(\alpha_{d}=0.95\right)$.

The demand curves for each interval are shown in Figure 9. Note that the intervals $[0,2]$ and $[0.5,1.5]$ allow greater deviations from the maximum demand, allowing higher generation values to be reached, especially for the case [0,2]. With regard to the H1 and $\mathrm{H} 4$ plants, the generation curves are very similar in the three cases analysed, showing only a small shift of the produced value. On the other hand, the generation curves for the $\mathrm{H} 2$ and $\mathrm{H} 3$ plants change significantly from one case to the other, showing greater flexibility in responding to demand.

The [0.95-1.05] case has resulted in a total gain of $\mathrm{R} \$ 5.8$ million, while the [0.5-1.5] case has increased to total gain to $\mathrm{R} \$ 10.6$ million, showing that there is a possibility of suggesting production curves that are more financially attractive. As the optimization is being carried out based on the demand values already approved by the ONS, the new proposed curves must also be feasible in the operating direction.

The inclusion of energy coming from a spot market allows the decision maker to save energy at cheaper price times and satisfy the contracted demand with energy coming 

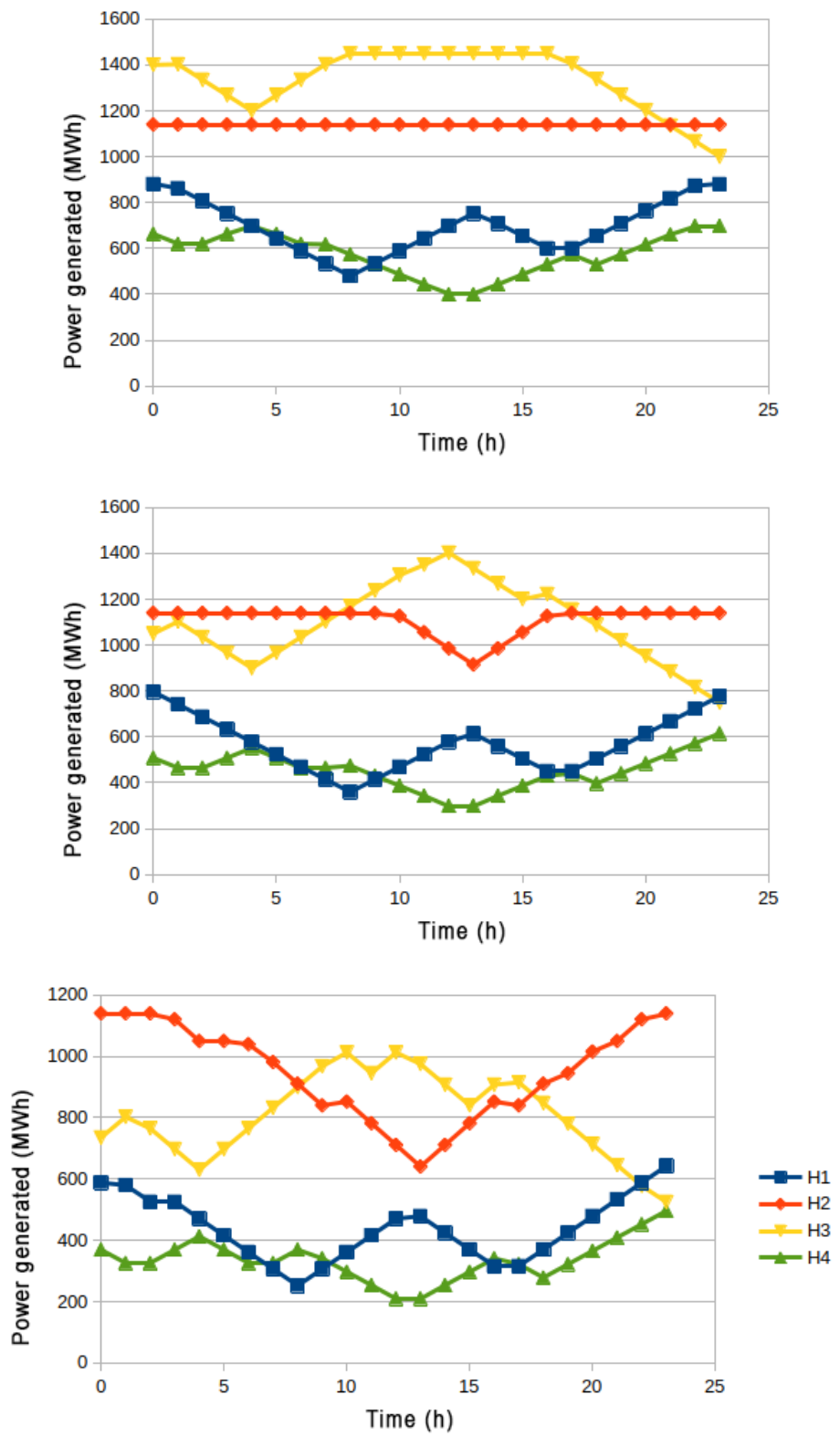

Figure 9. Power curves generated for each of the studied intervals. (Top) [0,2], (Centre) $[0.5,1.5]$, and (Bottom) [0.95,1.05].

from the spot market and use this saved volume to generate energy at times that are more financially attractive.

The generated power was distributed equally among the generated units, as can be seen in Figure 11. This behaviour is observed for the four reservoirs. 

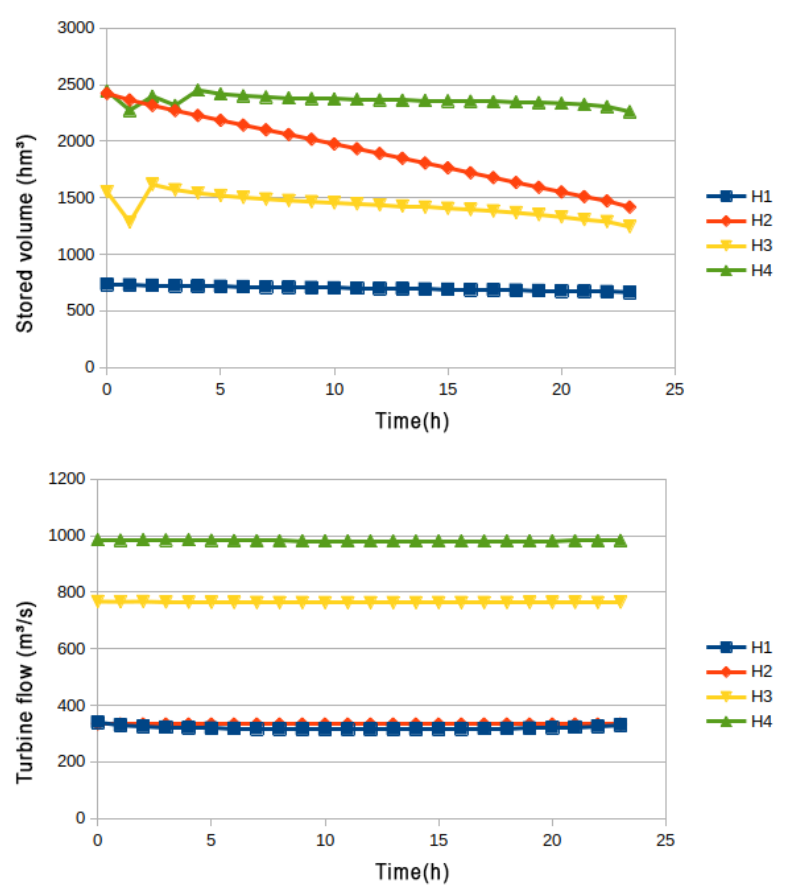

Figure 10. Reservoir volume and turbine flow for case [0.95,1.05].
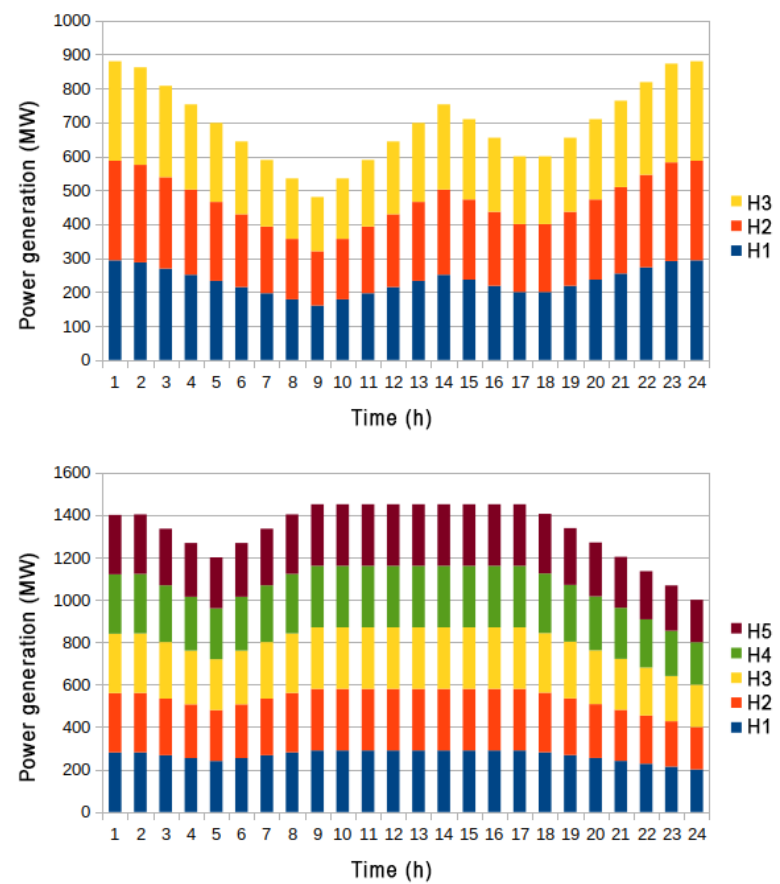

Figure 11. Power per turbine for reservoir H1 (top) and H5 (bottom). 


\subsection{Studies with the mixed-integer linear programming model}

The mixed-integer linear programming model was used with a different goal in mind. We took advantage of its capability of individually turning generators on and off to access possible advantages of using a few generation units or plants at higher power levels (and thus higher efficiencies) against using many units at lower power outputs. It is worth noting that in real-world situations there is a cost associated to the wear caused by the start-up processes, which was disregarded in the case study; yet, if the correct values are supplied, our model is capable of accounting for them.

Two different strategies were adopted to supply the same overall demand. In the first, each power plant had a quota of power to produce. In the second, the optimizer was free to change the power output of each plant, as long as the overall output was maintained.

Figure 12 shows the power demanded from each plant in the system at each time of the day, as well as the number of generators powered on in each plant. On the other hand, Figure 13 shows the same data but if a joint power quota for all plants.
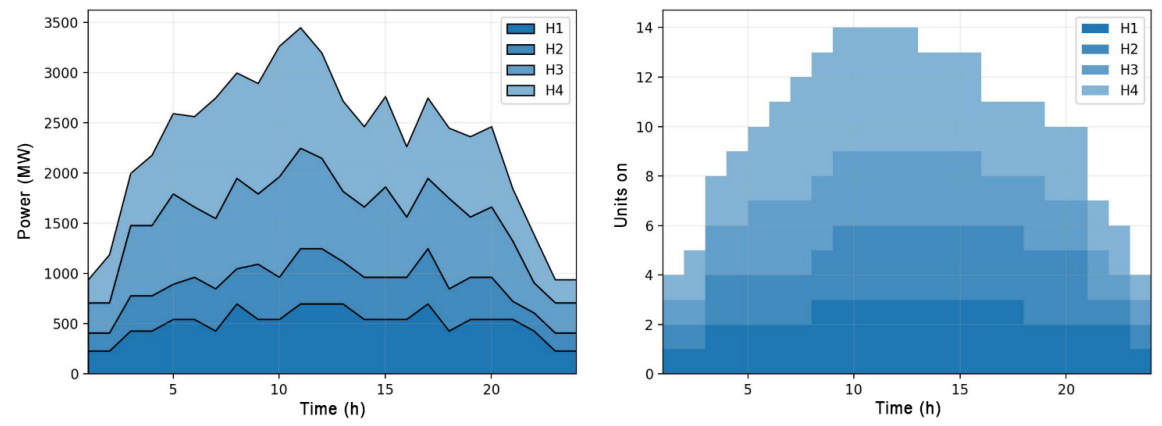

Figure 12. Power per plant and number of generating units on for an individual power quota.
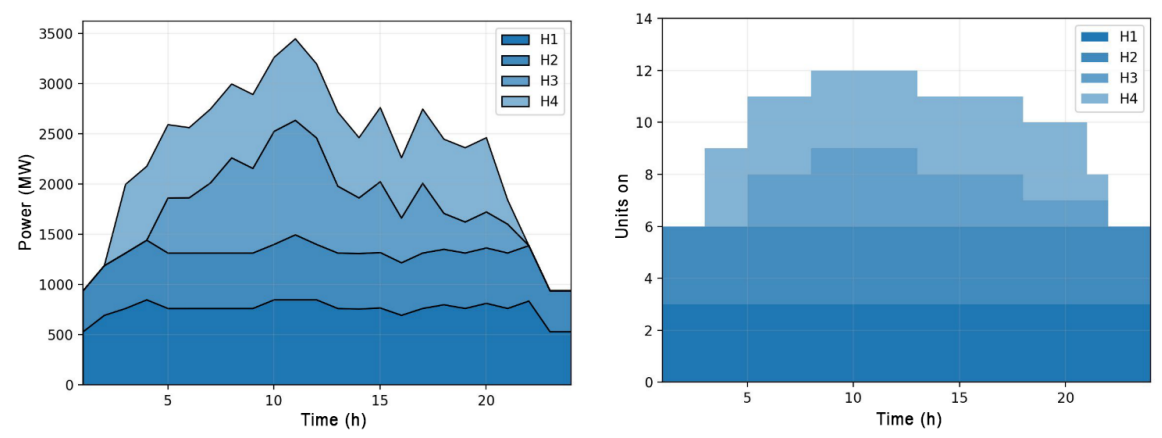

Figure 13. Power per plant and number of generating units on for a joint power quota.

In the scenario where each plant had its quota, the overall volume of water used was $52400 \mathrm{hm}^{3}$, which was reduced to $48500 \mathrm{hm}^{3}$ by using the joint quota, a $7.5 \%$ reduction due to the ability of choosing to use only the most efficient units at each moment. It is 
worth noting that this is an idealized scenario as in practice it would require fully turning on and off entire plants periodically; nonetheless, this analysis shows that there might be some room for improvement in this sense.

\section{Conclusion}

Both types of modelling shown in this study have shown potential strategies for improving the overall gain of a system of power plants. The non-linear approach was able to suggest power production curves that optimize the gains of the plant operators. On the other hand, the mixed-integer linear approach was able to suggest more optimum distributions of power generation for each plant, which could produce the same amount of energy while using less water. In a world where new types of sustainable energy sources are sought and implemented, strategies such as these might aid hydroelectric power plant operators to coexist with those new energy sources, choosing to generate power in a manner that maximizes their gain as well as minimizes the use of water resources.

It is important to observe that the CPU time for running both approaches was a few minutes. This is a very welcome feature, as one of the possible applications is to run simulations within one hour in order to suggest more profitable demand curves for the next day.

As a suggestion for continuation of this work, we would like to point out the following approaches:

- Use of other optimization libraries, such as IPOPT ${ }^{1}$, which would allow using the original non-linear equations instead of their simplifications.

- Comparison of the solution found by the non-linear approach and the one found using linearisation and a mixed-integer linear programming model.

- Addition of "prohibited zones" using non-linear restrictions of the type

$$
\left(h_{j r t}-\hat{H}_{j}\right)^{2}+\left(q_{j r t}-\hat{Q}_{j}\right)^{2} \geq \hat{R}_{j},
$$

where $\hat{H}_{j}$ e $\hat{Q}_{j}$ would be prohibited values of height and flow, $\hat{R}_{j}$ would be a suitable radius the encompasses the prohibited region. There are other forms of modelling, such as using the infinity norm and modelling using the $\max \{0, \cdot\}^{2}$ function, with the disadvantage of loss of gradient continuity.

\section{References}

[1]Erlon Cristian Finardi and Murilo Reolon Scuzziato. "Hydro unit commitment and loading problem for day-ahead operation planning problem". In: International Journal of Electrical Power 6 Energy Systems 44.1 (2013), pp. 7-16. ISSN: 0142-0615. DOI: https : / / doi org/10.1016/j . ijepes . 2012.07 .023. URL: https : / / www . sciencedirect.com/science/article/pii/S0142061512003833.

[2]EPE. Hidrelétricas. Disponível: https://www.epe.gov.br/pt/areas-de-atuacao/ energia-eletrica/expansao-da-geracao/fontes. Acesso: 25/03/2021. Dec. 2017.

1 https://coin-or.github.io/Ipopt/ 
[3]Carlos Augusto Machado Gomes. "Otimização da operação diária de usinas hidrelétricas em cascata utilizando metaheurísticas". MA thesis. 2018, p. 122.

[4]Murilo Reolon Scuzziato. "Proposta de um modelo para alocação ótima de unidades hidrelétricas para usinas em cascata". MA thesis. 2011, p. 111.

[5]Raouia Taktak and Claudia D'Ambrosio. "An overview on mathematical programming approaches for the deterministic unit commitment problem in hydro valleys". In: Energy Systems 8.1 (2017), pp. 57-79. 